\title{
Nutraceutical Properties of the Green Tea Polyphenols
}

Mudasir Ahmad ${ }^{1}$, Waqas N Baba ${ }^{1}$, Umar Shah¹, Asir Gani ${ }^{2}$, Adil Gani' ${ }^{*}$ and Masoodi FA ${ }^{1}$

${ }^{1}$ Department of Food Science and Technology, University of Kashmir, Srinagar, India

${ }^{2}$ Sant longowal Institute of Engineering and Technology, Punjab, India

\begin{abstract}
Green tea is manufactured from the leaves of the plant Camellia sinensis and has been regarded to possess anti-cancer, anti-obesity, anti-atherosclerotic, antidiabetic and antimicrobial effects. The main bioactive components present in green tea are polysaccharides, flavonoids, vitamins $B$, catechin compounds, fluoride and etc. Among them catechin compounds have been proven to have a variety of physiological functions. Due to the popularity of the health benefits of tea catechins, new products have been developed with tea as an active ingredient such as ready-to-drink (RTD) tea beverages, confections, ice creams, cereal bars and pet foods. This review summarizes several lines of evidence to indicate the health-promoting properties of green tea and their potential to be used in development of foods with added nutraceutical value.
\end{abstract}

Keywords: Tea polyphenols; Anticancer; Antidiabetic; Antimicrobial and intestinal digestion

\section{Introduction}

Camellia sinensis is one of the health promoting tea plants belonging to the family of Theaceae [1]. Tea plant grows in a form of an evergreen shrub in areas with suitable cultivation conditions (optimal temperature in the range of $15-20^{\circ} \mathrm{C}$ [2]. Tea leaves are manually harvested after each flush (the sprouting of the top two leaves and bud) and then processed. Leaves of the tea plant are rolled, chopped and dried. Dried leaves when added to boiling water yield an extract or tea beverage. Tea was originally discovered when tea leaves fell into boiling water at a reception for Chinese Emperor Shen-Nung about 2735 B.C. The difference between different varieties of tea is due to the difference in processing methods. The processing steps that are commonly used are withering, rolling, oxidation and drying. Green tea does not undergo oxidation and is steamed to oxidation making it distinctive in color and taste from black and oolong tea ("Andao," n.d.). Green tea resembles the original, untreated tea leaf in aromas and flavors. Oolong tea is also known as partially oxidized tea. The range of oxidation lies between $7.5 \%$ (slight oxidation) to $70 \%$ (complete oxidation). Black tea is completely oxidized. The dark color of black tea is due to the phenolase catalyzed oxidation of catechins in green tea [3]. An enzyme, polyphenol oxidase, is activated during the rolling and chopping process of the tea leaves. People in China have heated the leaves of the Camellia sinensis plant to inactivate the polyphenol oxidase which has resulted in green tea. Oxidation of polyphenol oxidase for about 1 or 2 hours results in oolong tea.

Tea is produced by processing shoots that include tender apical bud and subtending three leaves [4]. Infusion of leaves with hot/cold water results in a tea beverage. Processing procedures of the Chinese include roasting the tea shoots in a metal roaster and then using the unidirectional rotary roller for processing. The unidirectional roaster twists the leaves and compact the particles. The Japanese use steaming procedures to inactivate the shoots and then process by bi-directional rolling. Bi-directional rolling makes the shoot surface flat without any twists and by spreading the leaf juice over the entire surface.

The chemistry of tea is complex: polyphenols, alkaloids, amino acids, proteins, volatile compounds, minerals and trace elements $[5,6]$. The most abundant Green Tea Polyphenols (GTPs) are the Flavanolscatechin (C), epicatechin (EC), Gallocatechin (GC), Epigallocatechin (EGC), Catechin 3-O-Gallate (CG), Gallocatechin 3-O-Gallate (GCG), Epicatechin 3-O-Gallate (ECG) and
Epigallocatechin 3-O-Gallate (EGCG). But there are other compounds in green tea with interest for human health like fluoride, caffeine, minerals and trace elements like, chromium and manganese [7-9].

The aim of this review is to summarize the information related to beneficial properties of the green tea poly phenols and the application of green tea in food industry.

\section{Green tea polyphenols}

Polyphenols are powerful antioxidants and free radical scavengers. They are strong scavengers of superoxide, hydrogen peroxide, hydroxy radicals, and Nitric Oxide (NO) produced by various chemicals. Flavonoids are polyphenolic compounds that include the subclasses of flavanones, flavones, isoflavones, flavanols (flavans), flavonols and anthocyanins [10]. Green tea contains several groups of polyphenols that include flavonols (quercetin, kaempferol, and rutin, phenolic acids, theanine, flavor compounds, and leucoanthocyanins, accounting for up to $40 \%$ of the dry leaf weight [11]. The pharmaceutical activities of the components have been studied [12-14]. Chemically, the beverage is characterized by the major polyphenolic catechins such as (-)-epigallocatechin- 3-gallate (EGCG), (-)-epigallocatechin (EGC), (-)-epicatechin-3- gallate (ECG), and (-)-epicatechin (EC); these are the most abundant water-soluble components of tea $[11,15]$ Polyphenols are present at $10 \%$ to $15 \%$ in green tea and $5 \%$ in black tea. The polyphenols constitute about $42 \%$ of the dry weight of green tea extract, of which $26.7 \%$ comprise catechingallate components such as EGCG (11.16\%), ECG (2.25\%), EGC (10.32\%), epicatechin (2.45\%), and catechin $(0.53 \%)$. An infusion of green tea contains up to $200 \mathrm{mg}$ of catechins [16]. Green tea extracts have higher antioxidant capacity than black tea, and the total antioxidant potential correlates strongly with the total phenolic content of tea $[17,18]$. Shahidi and Alexander

*Corresonding author: Adil Gani, Department of Food Science and Technology, University of Kashmir, Srinagar, India, Tel: +91-8803023830; E-mail: adil.gani@gmail.com

Received July 21, 2014; Accepted October 28, 2014; Published November 06 , 2014

Citation: Ahmad M, Baba WN, Shah U, Gani A, Gani A, et al. (2014) Nutraceutical Properties of the Green Tea Polyphenols. J Food Process Technol 5: 390 doi:10.4172/2157-7110.1000390

Copyright: @ 2014 Ahmad M, et al. This is an open-access article distributed unde the terms of the Creative Commons Attribution License, which permits unrestricted use, distribution, and reproduction in any medium, provided the original author and source are credited. 
[19] found green tea catechins to inhibit the oxidation of meat lipids better than $a$-tocopherol and gallates of catechins. They can also chelate metal ions, often decreasing the metal ion pro-oxidant activity. Tea polyphenol scavenge the reactive oxygen species and chelate transition metal ions in a structure-dependent manner. The flavonoids are antioxidants by virtue of the number and arrangement of their phenolic hydroxyl groups [20]. The chemical structures contributing to the effective antioxidant activity of catechins include the vicinal dihydroxy or trihydroxy structure, which can chelate metal ions and prevent the generation of free radicals. The structure also allows for electron delocalization, conferring high reactivity to quench free radicals [21-23]. The antioxidant effect of polyphenols depends on their structure and the position as well as number of hydroxyl groups. EGCG has the greatest potential in scavenging free radicals, followed by ECG and EC, with EGC being the weakest [24]. Vitamin C is an anti-scorbutic, prevents cataracts, and strengthens the immune system. Some of the essential health benefiting effects of these polyphenols is discussed below:

\section{Anticancer properties}

One benefit of consuming green tea is that carcinogenesis in the digestive tract is postulated to be inhibited by ECGC as demonstrated in cells [25]. Okabe et al. showed that polyphenols from tea inhibited the growth and disintegration of a human stomach cancer cell line KATO III, and also inhibited tumor necrosis factor-a ( TNF-a) release from the cells. The order of polyphenols that followed the inhibition was ECG, EGCG, EGC and the aflavins. Inhibition of TNF -a release from a human stomach cancer cell line (KATO III) took place with the tea polyphenols (ECG, EGCG, EGC) treated with okadiac acid. EGCG was shown to prevent neuronal cell death caused by several neurotoxins [26]. Gastrointestinal tract cancer is mainly associated with an excess intake of protein and fat [27]. The polyphenols of green tea have been shown to exhibit inhibitory effects on cancer of the gastrointestinal tract and also have shown preventive effects against several other types of cancer. However, the evidence to support the preventive effects of green tea polyphenols on stomach and intestinal cancer is not clear. Studies indicate that green tea has a protective effect on adenomatous polyps and chronic atrophic gastritis formations. The inhibitory effect of green tea polyphenols was studied on the human lung cancer cell line, PC-9 [28]. Polyphenols that were examined included EGC, ECG, EGCG, and EC. Comparing their inhibitory effect, ECG and EGC showed the same potency as EGCG but EC showed less inhibitory effect. These studies shows the protective effects of green tea and health benefits associated with it on the cell line studied. Breast carcinoma is considered to be one of the most common cancers in women [29]. Breast cancer is more prevalent in western countries compared to Japan because of their daily intake of green tea as part of the diet. Consumption of green tea prior to the clinical cancer onset is believed to have decreased the risk of stage I and II breast cancer in women. Drinking green tea is believed to inhibit certain cancers, such as lung, skin, esophagus, liver, and stomach [30]. Tea catechins are mainly absorbed by the small intestines and are metabolized by enzymatic reactions. Epidemiological studies on the consumption of green tea and risks associated with cancer are still not clear. The reasons for the inconclusive studies might be due to poorly designed studies, differences in lifestyle and metabolic systems of individuals. Oxidative stress plays a major role in several liver diseases. Green tea has an antiproliferative activity on hepatoma cells, suppresses hepatoma-induced hyperlipidemia (hypercholesterolemia and hypertriglyceridemia), and also prevents hepatotoxicity [31]. Green tea may be a chemopreventive agent for hepatocarcinogenesis in the absence of chronic hepatocyte damage. It suppresses D-galactosamine induced liver injury in rats, which could be through inhibition of tumor necrosis factor-induced apoptosis [32]. Daily ingestion of green tea prevented hepatotoxicity (increase in serum glutamic-oxaloacetic transaminase and glutamicpyruvic transaminase; decrease in hepatic glycogen, serum triglyceride, and lactate dehydrogenase) and cell proliferation in the liver of rats on administration of 2-nitropropane [33].

Most pro-carcinogens require metabolic activation by metabolite enzymes such as phase I and II enzymes in order to convert to electrophiles before they can exert any carcinogenic effects [34]. In limiting the formation of carcinogens, green tea and its catechins promote the elimination of procarcinogens such as polycyclic hydrocarbons and heterocyclic amines from the body by inducing phase I cytochromes $\mathrm{P}_{450}$ $1 \mathrm{~A} 1,1 \mathrm{~A} 2$, and $2 \mathrm{~B} 1$ enzymes and phase II detoxification enzymes, for example, GT [35]. The procarcinogen-activating enzyme cytochrome P450 3A4 is also suppressed [36,37]. 2,3,7,8-Tetrachlorodibenzo-pdioxin (TCDD) is the most toxic compound of dioxin congeners [38]. Exposure of experimental animals to dioxins causes adverse effects such as body weight loss, immunosuppression, endocrine disruption, cancer promotion, teratogenesis, and lethality. Dioxins bind to the cytosolic aryl hydrocarbon receptor (AhR), commonly called the dioxin receptor, resulting in its transformation. Since AhR transformation is the initial step in the expression of dioxin toxicity, inhibition of transformation would protect humans from toxic effects. Theaflavins inhibit the binding of the TCDD to the AhR and also the binding of the transformed AhR to the specific DNA-binding site as putative mechanisms. Tea extracts can inhibit the cytochrome $\mathrm{P}_{450}$-mediated metabolism of 2-amino-3-methylimidazo, [4,5-f] quinoline (IQ) into its ultimate mutagenic metabolite forms, and interact with both the promutagens and their metabolites in a way that can reduce their mutagenic potential [39]. Benzopyrene (BaP)- and cyclophosphamide $(\mathrm{CP})$-induced genotoxicity in microbial and mammalian test systems are inhibited in a dose-dependent manner by theaflavins [40]. The polyphenols of black tea are more potent inhibitors of mutagenicity than those of green tea caused by the food mutagen PhIP $[41,42]$.

Cigarette smoking is a known cause of lung cancer and other respiratory diseases. Cigarette smoke contains numerous compounds that generate reactive oxygen species which can damage DNA directly or indirectly via inflammatory processes $[21,22,43]$. Regular drinking of green tea might protect smokers from oxidative damages and reduce cancer risk or other diseases caused by free radicals associated with smoking [44]. A tea infusion fed to rats in drinking water during an N-nitroso-methylbenzylamine (NMBA) adminisration period inhibited esophageal tumorigenesis $[45,46]$. Along with the antioxidative activity, green tea is shown to exhibit tumor suppressing activity in a cell [47].

\section{Hypocholestrolemic and other miscellaneous health benefiting effects}

In the book "Yojokun" published in the Edo period, Ekiken Kaibara described that according to the ancient Chinese medical doctor, long-term drinking of green tea would result in a lean body by removing body fat. Evidence has accumulated to show that the ingestion of green tea and tea catechins leads to a reduction in body fat as described in recent reviews [48-50]. Anti-atherosclerotic effects of catechins have also been reported. Muramatsu et al. [51] found that tea catechins decreased plasma total cholesterol, cholesterol ester, and total cholesterol- HDL-cholesterol (VIDL-DLDL-cholesterol) levels and lowered the atherogenic index (VLDL-DLDLcholesterol/HDLcholesterol), indicating that they exert a hypocholesterolemic effect, 
in cholesterol-fed rats. Catechins have been shown to prevent vascular smooth muscle cell invasion by inhibiting MT1-MMP activity and MMP-2 expression [52]. The ability of green tea to prevent cell invasion and matrix degradation might contribute to its protective effect on atherosclerosis and cancer.

Green tea has been known to prevent dental caries for decades. EGCG has received significant attention for its effects on inhibition of HIV infection and multidrug-resistant Staphylococcus aureus infections [53,54]. EGCG has been shown to inhibit HIV-1 replication by inhibiting HIV reverse transcriptase and by envelope. EGCG has been shown to prevent the attachment of the HIV-1 virion, gp120, to the CD4 molecules on T-helper cells, thus preventing the initial step in the HIV-1 infection process [55]. However, in vitro studies with pure N.T. Zaveri / Life Sciences 78 (2006) 2073-2080 2077 EGCG need to be interpreted with caution, since the stability of EGCG in cell culture media is questionable [56,57]. EGCG's polyphenolic nature and its high affinity for protein may also confound the results of in vitro experiments that use pure EGCG. Interestingly, EGCG and other galloyl-containing catechins were also identified in a high through put screening assay as inhibitors of scrapie-associated prion protein formation [58]. One study on irradiation used green tea polyphenols that were irradiated with dose of 40 Kilogrey's (kGy) more than the mass of the recommended level by the International Consultative Group of Food Irradiation (ICGFI) as a safe dose for better understanding the effect of irradiation. Irradiation of polyphenols increased the antimicrobial activities against Staphylococcus aureus and Streptococcus mutans. The study indicated that irradiation of green tea polyphenols maintains the biological activities and increases the anti-microbial activities [59]. Study also revealed the inhibition ranges for various bacterial (Escherichia coli, S. aureus, S. mutans) were 9.3, 10.1 and 9.3 $\mathrm{mm}$ in non-irradiated control but $10.8,11.0$ and $11.7 \mathrm{~mm}$ in irradiated samples, respectively. Thus irradiation positively changes the biological and anti-microbial activities of green tea samples.

A study was conducted to evaluate the inhibitory effects of grape seed extract, green tea extract, nisin and their combinations against Listeria monocytogenes [60]. The inhibitory effect was evaluated in a phosphate buffer solution (PBS) medium which contains $10^{9}$ colony forming units (CFU) of L. monocytogenes. Turkey frankfurters were inoculated by $10^{6} \mathrm{CFU} / \mathrm{g}$ of L. monocytogenes. The inoculated frankfurters with and without the addition of antimicrobial agents were dipped in soy protein forming soy solutions and stored at $4^{\circ} \mathrm{C}$ or $10^{\circ} \mathrm{C}$ [60]. Weekly for 28 days, the inhibitory effects of edible coatings were evaluated for frankfurters. Growth and recontamination of L. monocytogenesis shown to be controlled by an edible fat coating containing either grape seed extract or green tea extract in combination with nisin in ready-to-eat meat products.

\section{Green tea applications in milk beverages}

Tea catechins health benefits are not only associated with antioxidant activities but also include free metal chelation, scavenging of reactive oxygen and nitrogen species, as well as inhibition of lipoxygenase and cycloxygenase [61]. In most of the countries, tea is formulated with milk in different proportions to improve sensory properties; however, green tea is not traditionally consumed with milk even though formulations of green tea with soy and other dairy products are increasing. Studies show that consumption of tea with milk may have less availability of polyphenols, however, other studies have indicated that the addition of milk to black tea does not influence the absorption of tea catechins and its antioxidant activities [61]. Many methods have been developed for effective extraction of catechins in complex food formulations but methods were not suitable for milk based products. Strong catechin-protein interaction occurs in milk based products. Proteins have to be removed by a deproteinization step prior to the analysis so that this step can prevent precipitation onto the HPLC column during analysis. Various methods of deproteinization like pepsin treatment, methanol deprotenization and acid precipitation were studied. Total catechins were highly recovered by pepsin treatment (89-102\%) then by methanol deproteinization (78-87\%) and followed by acid precipitation (20-74\%) [61].

It is a common habit to add lemon to tea in United Kingdom, so a study was conducted to see the effect of lemon on the polyphenols of green tea. The study showed that the addition of lemon (ascorbic acid) increased the total antioxidant capacity linearly up to $30 \mathrm{mg}$ ascorbic acid/100 mL tea solution.

\section{Other green tea products}

Knowing the health benefits of tea catechins, new products have been developed with tea as an active ingredient in products such as Ready-To-Drink (RTD) tea beverages, confections, ice creams, cereal bars and pet foods. Some researchers have focused on the incorporation of Green Tea Extract (GTE) into foods, such as bread [62] cereals, biscuits and dairy products [63]. However, in addition to its bioactive components, green tea is also a rich source of other nutritional substances, such as dietary fiber and protein, which are usually wasted after tea polyphenols extraction [64]. Instead of drinking green tea some people apply green tea bags to their to soothe sunburn and prevent skin cancer due to sun exposure. Green tea bags are also used to decrease puffiness under the eyes as a compress for tired eyes or headache and to stop gums from bleeding after tooth is pulled. Green tea in candy is used for gum disease. Green tea is used in an ointment for genital warts. Drinking green tea with lemon improves the health benefits of the tea, according to researchers at Purdue University; Citrus Juice brings out green tea's anti-oxidants making them more available for your body to absorb. Catechins, which prefer the acid environment of the stomach, become degraded in more alkaline conditions of the small and large intestine, where nutrient absorption takes place. Lemon juice can increase the amount of catechins in body extracts from green tea by up to six times. Further compounds in tea can inhibit iron absorption from foods; drinking green tea with lemon reduces that effect. The combination of honey with green tea has been used since very past as they are very effective in healing wounds.

\section{Conclusion}

The above studies highlight the extensive health benefits of green tea. The bioactive components in green (EGCG, EGC, ECG and EC) possess antioxidant, anti-mutagenic, anti-diabetic, anti-inflammatory, anti-bacterial, and above all, cancer-preventive properties. Based on the above mentioned studies, it can be concluded that due to the purported health benefits of green tea, its consumption and applications have increased to large extent. The new products have been developed with tea as an active ingredient in products such as Ready-To-Drink (RTD) tea beverages, confections, ice creams, cereal bars and pet foods. This current study therefore paves way towards incorporation of green tea powder into other commonly consumed eatables for production of functional foods.

\section{References}

1. Weisburger JH (1996) Tea antioxidants and health. Handbook of antioxidants New York.

2. Li YH, Gu W, Ye S (2007) Expression and location of caffeine synthase in tea plants. Russian Journal of Plant Physiology 54: 698-701. 
3. Friedman M, Kim SY, Lee SJ, Han GP, Han JS, et al. (2005) Distribution ofcatechins, theaflavins, caffeine, and theobromine in 77 teas consumed in the United States. Journal of Food Science 70: 550-559.

4. Sharma V, Gulati A, Ravindranath SD (2005) Extractability of tea catechins as a function of maufacture procedure and temperature of infusion. Food Chemistry 93: $141-148$

5. Stagg GV, Millin DJ (1975) The nutritional and therupatic value of tea: A Review. Journal of Science of Food and Agriculture 26: 1439-1459.

6. Yang XR, Ye CX, Xu K, Jiang YM (2007) Simultaneous analysis of purine alkaloids and catechins in Camellia sinensis, Camellia ptilophylla and Camellia ssamicavar. kuchaby HPLC. Food Chemistry 100: 1132-1136.

7. Cabrera C, Giménez R, López MC (2003) Determination of tea components with antioxidant activity. J Agric Food Chem 51: 4427-4435.

8. Powell JJ, Burden TJ, Thompson RP (1998) In vitro mineral availability from digested tea: a rich dietary source of managanese. Analyst 123: 1721-1724.

9. Hope SJ, Daniel K, Gleason KL, Comber S, Nelson M, et al. (2006) Influence of tea drinking on manganese intake, manganese status and leucocyte expression of MnSOD and cytosolic amino peptidase. Eur J Clin Nutr 60: 1-8.

10. Li J, Jiang Y (2007) Litchi flavonoids: isolation, identification and biological activity. Molecules 12: 745-758.

11. Graham HN (1992) Green tea composition, consumption, and polyphenol chemistry. Prev Med 21: 334-350.

12. Han C (1997) Screening of anticarcinogenic ingredients in tea polyphenols. Cancer Lett 114: 153-158.

13. Johnson MK, Loo G (2000) Effects of epigallocatechin gallate and quercetin on oxidative damage to cellular DNA. Mutat Res 459: 211-218.

14. Zhu BT, Taneja N, Loder DP, Balentine DA, Conney AH (1998) Effects of tea polyphenols and flavonoids on liver microsomal glucuronidation of estradiol and estrone. J Steroid Biochem Mol Biol 64: 207-215.

15. Balentine DA, Wiseman SA, Bouwens LC (1997) The chemistry of tea flavonoids. Crit Rev Food Sci Nutr 37: 693-704.

16. Lakenbrink C, Lapczynski S, Maiwald B, Engelhardt UH (2000) Flavonoids and other polyphenols in consumer brews of tea and other caffeinated beverages. $J$ Agric Food Chem 48: 2848-2852.

17. Benzie IF, Szeto YT (1999) Total antioxidant capacity of teas by the ferric reducing/antioxidant power assay. J Agric Food Chem 47: 633-636.

18. Langley-Evans SC (2000) Antioxidant potential of green and black tea determined using the ferric reducing power (FRAP) assay. Int J Food Sci Nutr 51: 181-188.

19. Shahidi F, Alexander DM (1998) Green tea catechins as inhibitors of oxidation of meat lipids. Journal of Food Lipids 5: 125-133.

20. Rice-Evans CA, Miller NJ, Paganga G (1996) Structure-antioxidant activity relationships of flavonoids and phenolic acids. Free Radic Biol Med 20: 933-956.

21. Frenkel K, Donahue JM, Banerjee S (1988) Benzo(a)pyrene-induced oxidative DNA damage. A possible mechanism for promotion by complete carcinogen. Oxy-radicals in molecular biology and pathology .UCLA symposia on molecular and cellular biology. New York: Alan R. Liss 82: 509-524.

22. Wei L, Wei H, Frenkel K (1993) Sensitivity to tumor promotion of SENCAR and C57BL/6J mice correlates with oxidative events and DNA damage. Carcinogenesis 14: 841-847.

23. Yang CS, Maliakal P, Meng $X$ (2002) Inhibition of carcinogenesis by tea. Annu Rev Pharmacol Toxicol 42: 25-54.

24. Hu XF, Yang XQ, Liu KX (2001) Structure-activity relationship of tea polyphenols. Beijing, China: Beijing Science Press.

25. Okabe S, Ochiai Y, Aida M, Park K, Kim SJ, et al. (1999) Mechanistic aspects of green tea as a cancer preventive: effect of components on human stomach cancer cell lines. Jpn J Cancer Res 90: 733-739.

26. Reznichenko L, Amit T, Youdim MB, Mandel S (2005) Green tea polyphenol (-)-epigallocatechin-3-gallate induces neurorescue of long-term serum-deprived PC12 cells and promotes neurite outgrowth. J Neurochem 93: 1157-1167.

27. Borrelli F, Capasso R, Russo A, Ernst E (2004) Systematic review: green tea and gastrointestinal cancer risk. Aliment Pharmacol Ther 19: 497-510.
28. Okabe S, Suganuma M, Hayashi M, Sueoka E, Komori A et al. (1997) Mechanisms of growth inhibition of human lung cancer cell line, PC-9, by tea polyphenols. Jpn J Cancer Res 88: 639-643.

29. Nakachi K, Suemasu K, Suga K, Takeo T, Imai K, et al. (1998) Influence of drinking green tea on breast cancer malignancy among Japanese patients. Jpn J Cancer Res 89: 254-261.

30. Mandel S, Weinreb, Amit T, Youdim MBH (2004) Cell signaling pathways in the neuroprotective actions of green tea polyphenol (-) -epigallocatechin-3gallate: implications for neurodegerative diseases. Journal of Neurochemistry 88: 1555-1569

31. Crespy V, Williamson G (2004) A review of the health effects of green tea catechins in in vivo animal models. J Nutr 134: 3431S-3440S.

32. He P, Noda Y, Sugiyama K (2001) Green tea suppresses lipopolysaccharideinduced liver injury in d-galactosamine-sensitized rats. J Nutr 131: 1560-1567.

33. Sai K, Kai S, Umemura T, Tanimura A, Hasegawa R, et al. (1998) Protective effects of green tea on hepatotoxicity, oxidative DNA damage and cell proliferation in the rat liver induced by repeated oral administration of 2-nitropropane. Food Chem Toxicol 36: 1043-1051.

34. Conney AH (1982) Induction of microsomal enzymes by foreign chemicals and carcinogenesis by polycyclic aromatic hydrocarbons: G. H. A. Clowes Memorial Lecture. Cancer Res 42: 4875-4917.

35. Sohn OS, Surace A, Fiala ES, Richie JP Jr, Colosimo S, et al. (1994) Effects of green and black tea on hepatic xenobiotic metabolizing systems in the male F344 rat. Xenobiotica 24: 119-127.

36. Lin JK, Liang YC, Lin-Shiau SY (1999) Cancer chemoprevention by tea polyphenols through mitotic signal transduction blockade. Biochem Pharmacol 58: $911-915$

37. Muto S, Fujita K, Yamazaki Y, Kamataki T (2001) Inhibition by green tea catechins of metabolic activation of procarcinogens by human cytochrome P450. Mutat Res 479: 197-206.

38. Fukuda I, Sakane I, Yabushita Y, Sawamura S, Kanazawa K, et al. (2005) Black tea theaflavins suppress dioxin-induced transformation of the aryl hydrocarbon receptor. Biosci Biotechnol Biochem 69: 883-890.

39. Chen HY, Yen GC (1997) Possible mechanisms of antimutagens by various teas as judged by their effects on mutagenesis by 2-amino-3-methylimidazo[4,5-f] quinoline and benzo[a]pyrene. Mutat Res 393: 115-122.

40. Shukla Y, Arora A, Taneja P (2003) Antigenotoxic potential of certain dietary constituents. Teratogenesis, Carcinogenesis, and Mutagenesis Suppl 1: 323-335.

41. Apostolides Z, Balentine DA, Harbowy ME, Weisburger JH (1996) Inhibition of 2-amino-1-methyl-6-phenylimidazo[4,5-b]pyridine (PhIP) mutagenicity by black and green tea extracts and polyphenols. Mutat Res 359: 159-163.

42. Apostolides Z, Balentine DA, Harbowy ME, Hara Y, Weisburger JH (1997) Inhibition of PhIP mutagenicity by catechins, and by theaflavins and gallate esters. Mutat Res 389: 167-172.

43. Hecht SS (1999) Tobacco smoke carcinogens and lung cancer. J Natl Cance Inst 91: 1194-1210.

44. Hakim IA, Harris RB, Brown S, Chow HH, Wiseman S, et al. (2003) Effect of increased tea consumption on oxidative DNA damage among smokers: a randomized controlled study. J Nutr 133: 3303S-3309S.

45. Han C, Xu Y (1990) The effect of Chinese tea on occurrence of esophageal tumo induced by $\mathrm{N}$-nitrosomethylbenzylamine in rats. Biomed Environ Sci 3: 35-42

46. Chen J (1992) The effects of Chinese tea on the occurrence of esophagea tumors induced by N-nitrosomethylbenzylamine in rats. Prev Med 21: 385-391.

47. Li HC, Yashiki S, Sonoda J, Lou H, Ghosh SK, et al. (2000) Green tea polyphenols induce apoptosis in vitro in peripheral blood T lymphocytes of adult T-cell leukemia patients. Jpn J Cancer Res 91: 34-40.

48. Chacko SM1, Thambi PT, Kuttan R, Nishigaki I (2010) Beneficial effects of green tea: a literature review. Chin Med 5: 13

49. Thavanesan $N$ (2011) The putative effects of green tea on body fat: an evaluation of the evidence and a review of the potential mechanisms. $\mathrm{Br} \mathrm{J}$ Nutr 106: 1297-1309.

50. Rains TM, Agarwal S, Maki KC (2011) Antiobesity effects of green tea catechins: a mechanistic review. J Nutr Biochem 22: 1-7. 
Citation: Ahmad M, Baba WN, Shah U, Gani A, Gani A, et al. (2014) Nutraceutical Properties of the Green Tea Polyphenols. J Food Process Technol 5: 390. doi:10.4172/2157-7110.1000390

51. Muramatsu K, Fukuyo M, Hara $Y$ (1986) Effect of green tea catechins on plasma cholesterol level in cholesterol-fed rats. J Nutr Sci Vitaminol (Tokyo) 32: 613-622.

52. El Bedoui J, Oak MH, Anglard P, Schini-Kerth VB (2005) Catechins prevent vascular smooth muscle cell invasion by inhibiting MT1-MMP activity and MMP2 expression. Cardiovasc Res 67: 317-325.

53. Nance CL, Shearer WT (2003) Is green tea good for HIV-1 infection? J Allergy Clin Immunol 112: 851-853.

54. Stapleton PD, Shah S, Anderson JC, Hara Y, Hamilton-Miller JM, et al. (2004) Modulation of beta-lactam resistance in Staphylococcus aureus by catechins and gallates. Int J Antimicrob Agents 23: 462-467.

55. Kawai K, Tsuno NH, Kitayama J, Okaji Y, Yazawa K, et al. (2003) Epigallocatechin gallate, the main component of tea polyphenol, binds to CD4 and interferes with gp120 binding. J Allergy Clin Immunol 112: 951-957.

56. Hong J, Lu H, Meng X, Ryu JH, Hara Y, et al. (2002) Stability, cellular uptake, biotransformation, and efflux of tea polyphenol (-)-epigallocatechin-3-gallate in HT-29 human colon adenocarcinoma cells. Cancer Res 62: 7241-7246.

57. Naasani I, Oh-Hashi F, Oh-Hara T, Feng WY, Johnston J, et al. (2003) Blocking telomerase by dietary polyphenols is a major mechanism for limiting the growth of human cancer cells in vitro and in vivo. Cancer Res 63: 824-830.
58. Kocisko DA, Baron GS, Rubenstein R, Chen J, Kuizon S, et al. (2003) New inhibitors of scrapie-associated prion protein formation in a library of 2000 drugs and natural products. J Virol 77: 10288-10294.

59. An BJ, Kwak JH, Son JH, Park JM, Lee JY, et al. (2005) Physiological activity of irradiated green tea polyphenol on the human skin. Am J Chin Med 33: 535-546.

60. Theivendran S, Hettiarachchy NS, Johnson MG (2006) Inhibition of listeria monocytogenes by nisin combined with grape seed extract or green tea extract in soy protein film coated on turkey frankfurters. Journal of Food Science 71: 39-44.

61. Ferruzzi MG, Green RJ (2006) Analysis of catechins from milk-tea beverages by enzyme assisted extraction followed by high performance liquid chromatography. Food Chemistry 99: 484-491.

62. Wang R, Zhou W (2004) Stability of tea catechins in the breadmaking process J Agric Food Chem 52: 8224-8229.

63. Sharma A, Zhou W (2011) A stability study of green tea catechins during the biscuit making process. Food Chemistry 126: 568-573.

64. Lu TM, Lee CC, Maud JL, Lin SD (2010) Quality and antioxidant property of green tea sponge cake. Food Chemistry 119: 1090-1095. 